\title{
Deterministic measurement and correction of the pad shape in full-aperture polishing processes
}

\section{Liao}

Q. Zhang

zhangqh502@sina.com

\section{R. Xie}

X. Chen

\section{S. Zhao}

\section{J. Wang}

Chengdu Fine Optical Engineering Research Centre, Chengdu, 610041, China

Chengdu Fine Optical Engineering Research Centre, Chengdu, 610041, China

Chengdu Fine Optical Engineering Research Centre, Chengdu, 610041, China

Chengdu Fine Optical Engineering Research Centre, Chengdu, 610041, China

Chengdu Fine Optical Engineering Research Centre, Chengdu, 610041, China

Chengdu Fine Optical Engineering Research Centre, Chengdu, 610041, China

Full-aperture polishing is a significant process in fabricating large optical flats because it restrains Mid-Spatial Frequency errors and removes material quickly on the whole optic surface. Nevertheless, optical flats fabricated by full-aperture polishing generally fail to meet the stringent requirement of surface figure, which has to be corrected by sub-aperture polishing processes. Surface figure of optical flats in full-aperture polishing processes is primarily dependent on the pressure distribution uniformity which correlates intensively with the lap shape. At present, practical and precise means are urgently desired for measuring and correcting the lap shape, especially the polyurethane pad lap. In the study, we present a novel method for deterministic measurement of the pad shape. The method obtains the height of the pad at spirally distributed locations implemented by the revolution of the pad and translation of the laser displacement sensor. The pad shape in terms of matrixes whose elements representing the heights at the corresponding locations is then calculated by interpolation algorithm based on the obtained data. Further, we propose a method for deterministic correction of the pad shape utilizing a small conditioning tool. The dwell time algorithm and implementation strategy for the dwell time are provided for common full-aperture polishers. These solutions for the deterministic measurement and correction of the pad shape have been validated on a full-aperture polisher with polyurethane pad. The polishing experiments revealed that the optic surface figure was obviously improved.

[DOI: http://dx.doi.org/10.2971/jeos.2015.15049]

Keywords: Optical flats, full-aperture polishing, pad shape, deterministic measurement and correction

\section{INTRODUCTION}

Large optical flats are key components used in interferometers, high-power lasers, telescopes and lithography lens. These optics are required to be with high precision of surface figure. In recent years, mid-spatial frequency (MSF) errors attract increasing attention, which have a significant influence on near-field modulation in high-power lasers and give rise to structured image artifacts in some optical systems [1]-[4]. Fabrication of high precision optical flats typically involves a series of steps including (1) shaping, (2) grinding, (3) fullaperture polishing, and (4) sub-aperture polishing [5]. The full-aperture polishing utilizes a large lap which commonly covers the whole optic surface through the polishing process, thus it has obvious advantage in restraint of the MSF errors (i.e. waviness) [6]. However, optical flats fabricated by fullaperture polishing processes hardly meet the specification of surface figure, which has to be subsequently corrected with sub-aperture polishing processes. Sub-aperture polishing processes are characterized by a small tool whose dwell time map on the optic is implemented by computer control, thus they are also known as computer controlled optical surfacing (CCOS). Various CCOS processes have been developed since the 1970s, such as small tool computer controlled polishing [7, 8], Magnetorheological Finishing [9], ion beam finishing [10], and so forth [11, 12]. These processes have brought great promotion of surface figure. Nevertheless, the tool influence function stroking on the optic for correction of local figure error inevitably results in waviness on the surface and hence deteriorates the mid-spatial frequency error (MSF). Thus, promoting the full-aperture polishing processes to get a much higher precision surface figure of optical flats will bring several advantages. Under this condition, material removal in CCOS will be greatly reduced, and hence the mid-spatial frequency error introduced in CCOS will be restrained. Further, the polishing time needed for a much higher precision optical flat will be greatly decreased.

The conventional full-aperture polisher is characterized by an annular lap, which consists of a pitch lap fixed on a base 


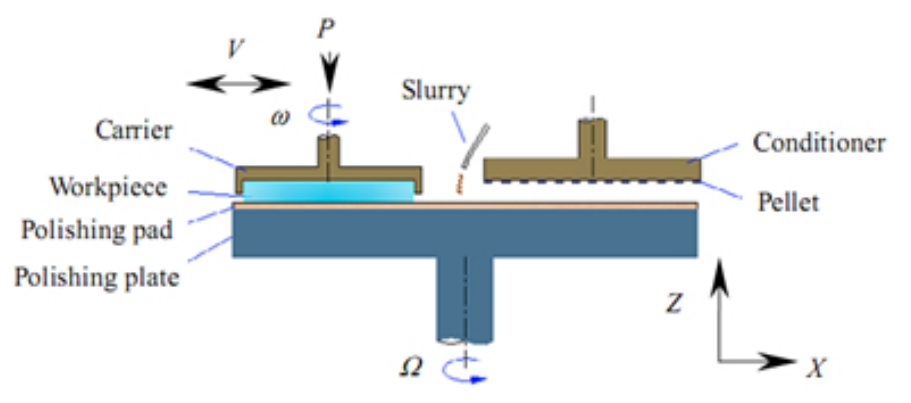

FIG. 1 Schematic of a typical full-aperture polisher with a polyurethane pad.

platen. The introduction of hyper-elastic polyurethane pad as a substitution for pitch makes full-aperture polishing easy to use high speeds and high pressures (see Figure 1), and hence yields a higher removal rate without concern for flow in the pitch $[13,14]$. During full-aperture pad polishing, the optic is rotated while being placed on the rotating pad covered with chemical slurries. Surface figure of the optic is determined by material removal distribution. The Preston equation empirically found by Preston in 1927 describes material removal rate $(M R R)$ of optic during polishing $[15,16]$ :

$$
\operatorname{MRR}(x, y, t)=k p(x, y, t) v(x, y, t)
$$

Where MRR is the local thickness removal rate, $p$ is the applied local pressure, $v$ is the instantaneous relative velocity of the polishing particle relative to the optic, and the microscopic and molecular level effects are described macroscopically by the Preston coefficient $(k)$. It predicts that $M R R$ is proportional to the polishing velocity and the applied pressure. Under a certain polishing condition, Preston coefficient is assumed to be constant in the entire optic/pad interface. Numerous researches have revealed that a uniform distribution of relative velocity can be obtained through the rotations of the optic and the platen at the same speed $[17,18]$. Therefore, the surface figure, to a great extent, depends on the variation of the pressure distribution on the optic surface [19]. As the optic is placed on the pad during polishing, the pressure distribution on the optic/lap interface is intensively correlative to the lap shape. In practical full-aperture polishing processes, flatness of the pad is always desired for promotion of the optic surface figure.

The planarity of the polyurethane pad has extensively been controlled by a large diamond protruded conditioner disc. Various researches revealed concavity of the pad shape by the conditioner disc oscillating on the pad [20, 21]. In summary, the disadvantages of these methods lie in: (1) There is no method for measuring the pad shape, which is usually speculated based on the surface figure of the polished optics; (2) The planarization of the polyurethane pad by conditioner disc oscillating on the pad is difficult to achieve by the large diamond conditioner disc.

This work develops systematic solutions to measure the pad shape and then to planarize it. In Section 2, a novel measuring method for the pad shape is proposed, and the configurations and procedures are detailed. In Section 3, a small conditioning tool is introduced to generate a wear function of the polyurethane pad, and then the optimal dwell time map of the tool which can be easily implemented by common full-

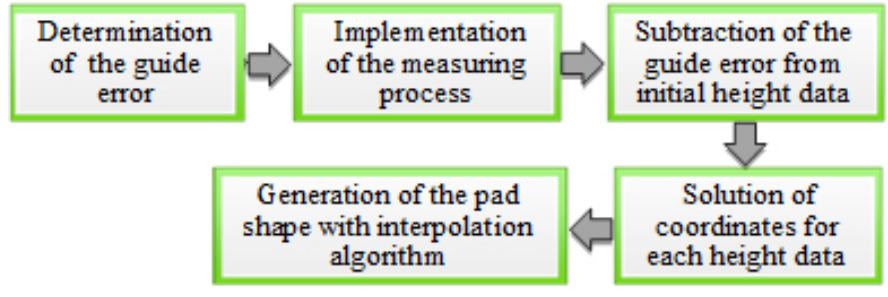

FIG. 2 Flow chart of measuring the pad shape.

aperture polisher is provided. In Section 4, the measuring and planarizing methods are validated by experiments conducted on a $\Phi 1.1 \mathrm{~m}$ polyurethane pad polisher.

\section{DETERMINISTIC MEASUREMENT OF THE PAD SHAPE}

The pad shape is measured by a laser displacement sensor. During measuring, the sensor is moving along the radial direction of the pad while the pad is rotating. Thus the height of the pad relative to the sensor, at the spirally distributed points, is obtained. In computer, the pad shape is described in terms of matrix, of which the elements represent the heights at uniformly distributed points. Herein, we calculate the height matrix by interpolation algorithm based on the detected data. Flow chart of measuring the pad shape is shown in Figure 2. In the following, the configuration and procedure for measuring and generating the pad shape are detailed.

\subsection{Configuration and preparation}

In the proposed method, a servo motor system is required of the full-aperture polisher to control the angular position and rotation rate of the base platen. Further, the translation stroke of the sensor has to cover the radius of the pad, and the position and linear velocity is required to be precisely controlled while the sensor is moving along the radial direction of the pad. In some full-aperture polishers, a workpiece spindle being able to oscillate above the pad is equipped to drive the optic through a holder, and then the sensor can be fixed on the holder. For the full-aperture polishers without such equipment, we present an auxiliary system for assisting the measuring procedure. As shown in Figure 3, a guide with straightness error less than $20 \mathrm{um}$ is installed above the pad at a height of about $2 \mathrm{~m}$. The guide is supported by two upright columns placed at each side of the polisher. The motor stator is fixed at one end of the guide. A movable block is connected to the motor rotor, and it is fastened on the sliders which are restricted by the guide. A servo system for the motor rotor controls the position and linear velocity of the movable block. The precision and stability of the guide plays an important role on measuring the pad shape.

The laser displacement sensor system consists of the sensor, data line and PC. The sensor is fixed on the movable block with the detecting point orienting to the pad surface. The distance between the sensor and the pad can be detected by the sensor. The detected data (i.e., the height of the sensor relative to the pad) is instantaneously transported to the PC by 


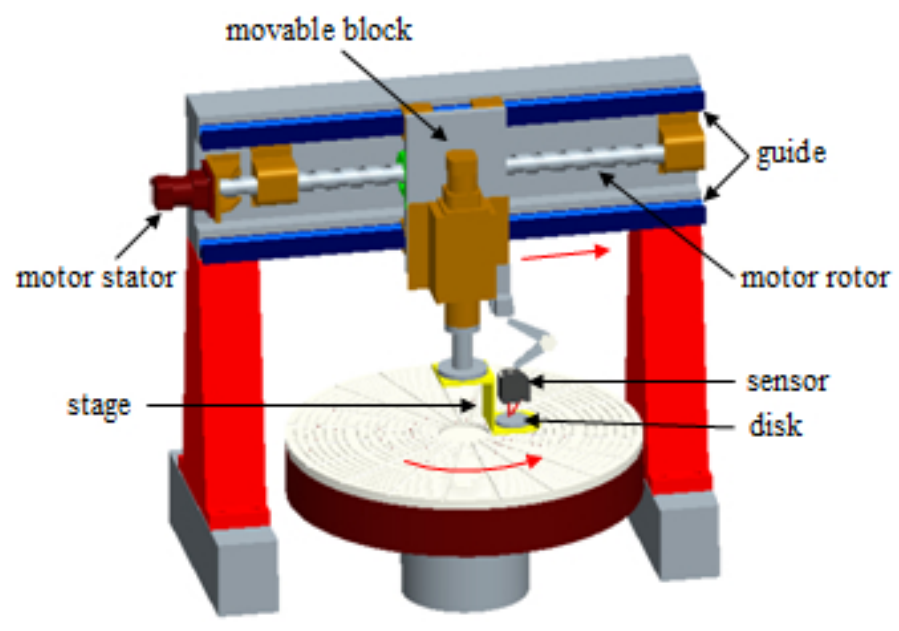

FIG. 3 Equipment and its configuration developed for measuring the pad shape.

the data line, which will be stored and processed in PC. The sensor has a resolution of $0.1 \mathrm{um}$, such as Keyence LK5000.

The motion errors of the sensor as it translates across the pad surface are interpreted as shape error in the pad. These errors can be measured and compensated for in the final calculations since they are generally related to manufacturing tolerances or errors in the guide geometry (i.e., guide error). Any variance of the guide error will be interpreted as the variance of the pad shape, thus the repeatability should be strictly restricted (e.g. $<0.4 \mathrm{um}$ ). The errors can be determined by using a high precision mirror $(\mathrm{PV}<1 \mu \mathrm{m})$ and an adjustable platform. Flatness error of the reference mirror is also included into the measuring data of the pad shape, thus the backside of the reference mirror is required of a high flatness, preferably better than $1 \mathrm{um}$. For determination of the guide error, the adjustable platform is mounted on the pad, while the mirror is placed at the platform with the high precision surface orientating above. The platform is equipped with several thimbles which can adjust the obliquity of the platform. In order to measure the guide error, the distance of the sensor and the mirror is desired to keep stable as the platen rotates, that is, the surface of the mirror and the spindle of the platen is identically vertical.

After the mirror is adjusted to be vertical to the spindle of the platen, then the guide error can be measured. The block is first moved to one end of the guide, and the detecting point of the sensor is settled at the mirror surface, which can guarantee a longest measuring distance. Then, the block driven by the verso motor is started to move along the guide at a constant liner velocity, driving the detecting point moving along the radial direction. The height of the sensor along the guide $e=f(x)$ (inner radius $\leq x \leq$ outer radius) is transported to the PC through the data line. This height distribution is the initial error of the guide, which has to be eliminated while measuring the actual pad shape.

\subsection{Measuring Procedure and Pad Shape Generation}

The angular rate of the pad and linear velocity of the movable block have a decisive effect on the measuring time and sam- pling spacing. A too large angular rate of the pad will result in wobbling of the plate, while a too small one will increase the measuring time, thus the angular rate of the pad is generally set within $5 \sim 10 \mathrm{rpm}$. As the sensor measures the pad in a spiral path, the linear velocity of the sensor determines the screw pitch along the radial direction. Generally a screw pitch of $3 \sim 8 \mathrm{~mm}$ would be appropriate. As the pad and sensor are specified of an optimal angular rate and linear velocity, then they are started to rotate around the spindle and move along the guide, respectively. The height of the pad at the detecting point is measured by the sensor which is fixed on the movable block or the holder, and the height data is simultaneously exported to the PC and then recorded. The kinematical configuration presents a spiral distribution of the detecting point on the pad surface, as shown in Figure 4 . We can build a rectangular coordinate system on the pad. The center is set at the rotating center of the pad, and the $X$ axis orients parallel to the above guide while going through the pad center. It is important that the detecting point starts at the inner radius for the annular pad or the geometric center for the circular pad. The coordinates of the detecting points can be obtained by:

$$
\left\{\begin{array}{l}
x(t)=\left(r_{0}+v t\right) \cos w t \\
y(t)=\left(r_{0}+v t\right) \sin w t
\end{array}\right.
$$

The height detected by the sensor is a function of measuring time, i.e., $h(t)$. The actual height of each position is the detected height minus the initial error of the guide at the corresponding position, as shown by Eq. (3). $f(x(t))$ represents the error of the guide at the position where the block is moving through at the measuring time $t$.

$$
z(t)=h(t)-f(x(t))
$$

Generally, the sensor records the height at a certain sampling frequency (e.g. sampling period $\mathrm{s}=10 \mathrm{~ms}$ ). Then the $\mathrm{x}$ and $\mathrm{y}$ coordinates and heights at the detecting positions can be obtained by:

$$
\left\{\begin{array}{l}
x(i)=\left(r_{0}+i \cdot s v\right) \cos (i \cdot s w) \\
y(i)=\left(r_{0}+i \cdot s v\right) \sin (i \cdot s w) \\
z(i)=h(i)-f\left(x_{i}\right)
\end{array}\right.
$$

Where $i=1,2, \ldots, n$ representing a series of detecting points.

The texture and structure of the pad surface has a great influence on the measuring accuracy. Generally, the polyurethane pad surface has numerous independent pores [22, 23]. The pores are separated by polyurethane wall structures that contain micron-sized asperities. Furthermore, polyurethane pads usually contain grooves designed to transport slurry. The sensor detects the height by a small laser beam ( $\Phi 25 \mathrm{um}$ ) which shots at the target surface. As the detecting point move on the pad surface, it may locate at the asperities, grooves, and pores. We introduced a measurement stage having a circular disk that rides on the surface of the pad as a means to bridge these small discontinuities on the pad surface and to provide physical averaging of the pad surface (see Figure 5). The measurement stage has a circular opening to accommodate the disk. The disk is constrained to move with the stage, but free to follow the vertical contour of the pad surface. Thus, the local height of the pad can be obtained by measuring the vertical position of the disk which provides a constant offset 

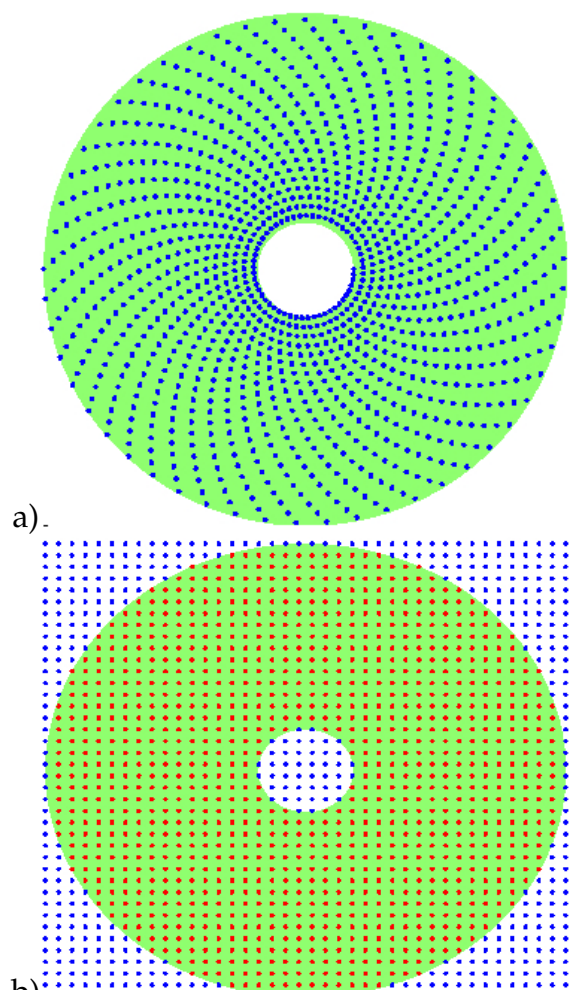

b)

FIG. 4 Schematic of distribution of the data points, (a) spirally distributed detecting points, (b) uniformly discretized points.

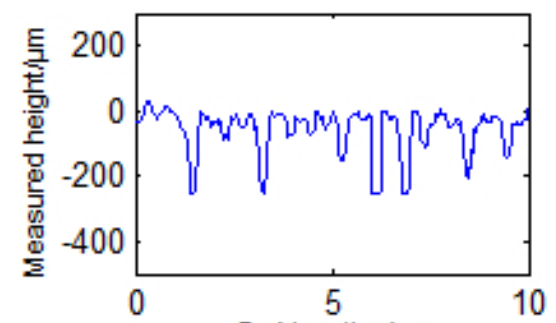

a)

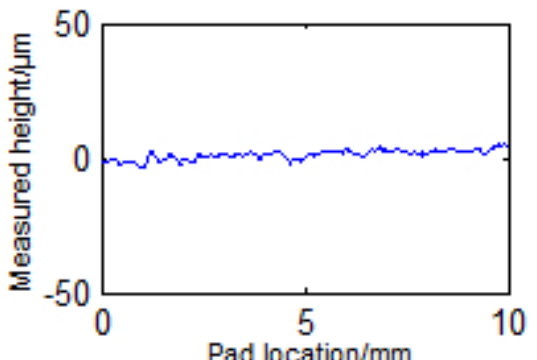

b)

Pad location/mm

FIG. 5 Profile of the pad along the spiral path, (a) measured without the circular disk, (b) measured with the circular disk.

to the pad. The main factor affecting the measurement precision is the stability of the disk as it translates across the pad surface. Tremble of the disk would bring with random errors. In practical measurement, the disk is configured with an appropriate size (e.g., $\geq \Phi 80 \times 20 \mathrm{~mm}$ ) so as to maintain contact with the pad.

The shape data are usually stored in matrix, which requires the sampling points are uniformly distributed in both $x$ and $y$ directions. To achieve it, the representative positions $\left(x^{\prime}(k, l), y^{\prime}(k, l)\right)$ are generated by discretizing the zone $(-r \leq x, y \leq r)$ with the same interval $\mathrm{d}$, as shown in

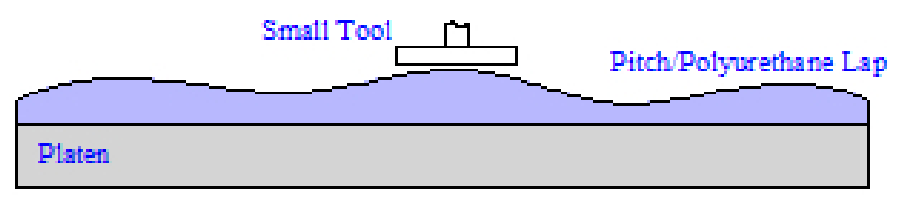

FIG. 6 Schematic of the deterministic correction of the pad shape with small tool.

Eqs. (5)-(6). Then, the heights at the representative positions within the pad surface can be solved by interpolation algorithm based on the heights of the detected points. The interpolating algorithm recommended here includes spline interpolant, shape-preserving interpolant, linear, quadratic, and so on. While the heights at the positions outside the pad zone are set to an invalid value. By doing this, we can obtain uniformly distributed positions and the corresponding heights, namely, $(x(i, j), y(i, j), z(i, j))$.

$$
\begin{aligned}
& \left\{\begin{array}{l}
x^{\prime}(k, l)=-r+l \cdot d \\
y^{\prime}(k, l)=-r+k \cdot d
\end{array} \quad k, l=0,1, \ldots, v ; \quad d=\frac{2 r}{v}\right.
\end{aligned}
$$

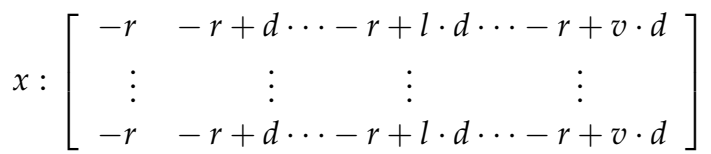

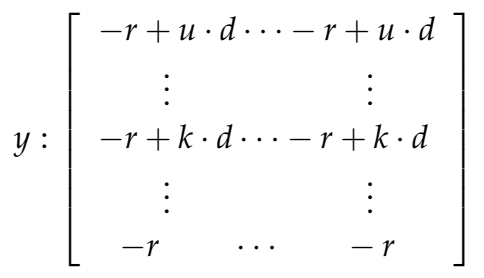

\section{DETERMINISTIC CORRECTION OF THE PAD SHAPE}

Herein, a novel method is proposed for correcting the shape error of the polyurethane pad in full-aperture polishing processes. We utilize featured small tools for generating small wear functions of the pad. By controlling the dwell time map of the tool on the pad (see Figure 6), the local error is corrected and hence the entire pad surface is planarized. In the following, the structure and configuration of the tool are detailed, and the dwell time algorithm and implementation strategy of the dwell time are also provided.

The polyurethane pad is a wear resistant super-elastic material [24]. Thus, the conditioning tool should be properly selected for a well-distributed wear function. The diamondimpregnated disc, which is extensively used for pad conditioning in chemical mechanical polishing processes, has been proven that it can regenerate a new pad surface for a fairly consistent removal and alter the pad shape so as to improve the global wafer flatness. Thus the diamond-impregnated disc of small size is an optimal selection to condition the pad locally and then obtain a desired wear function. We adopt a small diamond disc (e.g. $90 \mathrm{~mm}$ in diameter) as the tool, as shown in Figure 7 . The diamond disc is fabricated by electroplating, brazing or sintering a given amount of diamond grits into a side of a metal substrate. Diamond grits used have a size of 30-200 microns, and they are randomly arrayed on the 


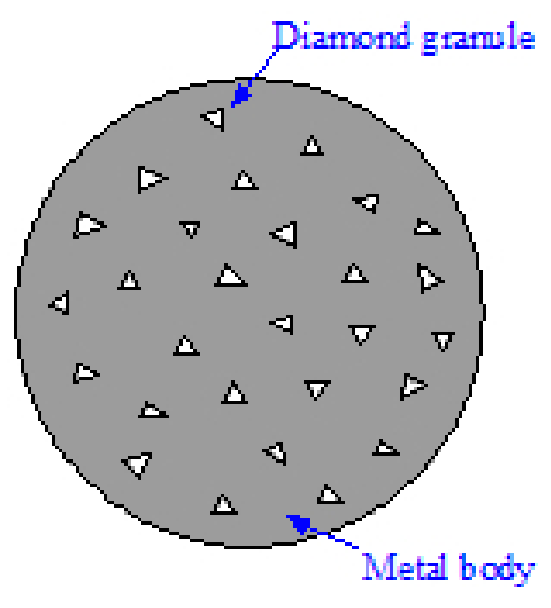

FIC. 7 Schematic of diamond-impregnated disc tool for conditioning the polyurethane pad.

substrate. The tool head is held and driven by the workpiece spindle, and the side with diamond grits is pressed against the pad surface.

The small tool is driven by the tool head, whose movement is controlled by the polisher through servo motors. The structure and kinetic configuration of the tool head have great influence on removal characteristic of the tool. Herein, we proposed a two-axis structure for the conditioning head, which is commonly utilized in computer controlled small-tool polishing process to obtain a near-Gaussian wear function (propitious to form correction). As shown in Figure 8, one rotating axis is set at the center of the diamond disc, and it is named self-rotating axis. The other rotating axis is set with an eccentric distance from the self-rotating axis. The diamond disc rotates about the both axes synchronously during pad conditioning. The 'static wear function' of the rotating tool (no translation on the pad) on the static pad can be determined by measuring the shape of pad before and after a certain period of conditioning. In order to correct the shape error of the pad, a dwell time map (i.e. tool ablation time as a function of tool position on the pad) is desired to achieve a target removal map. Dwell time at local sites of the pad is implemented by controlling the angular speed of the pad as well as the translation (along the pad radius) velocity of the tool. The 'dynamic wear function' during pad shape correction is required to be stable for an efficient correction process [25]. When the angular speed of the pad and translation velocity of the tool are much lower than the angular speeds of the tool, the kinematics is dominated by the angular speeds of the conditioner. Thus the 'dynamic wear function' approaches to the 'static wear function' and keeps stable during conditioning.

Accurate solution and implementation of the dwell time map on the pad is the key in realizing an efficient pad shape correction process. Spiral tool path is recommended considering the annular shape of the pad. Herein, we adopt 'uniform-areaincrement' type spiral path [26], in which the area enclosed by each dwell point is constant as shown in Figure 9. This tool path generates a series of uniformly distributed dwell points, and thus improves the movement stability of the polisher during implementing the dwell time. The dwell points in the polar coordinate can be obtained by Eq. (7), in which the first

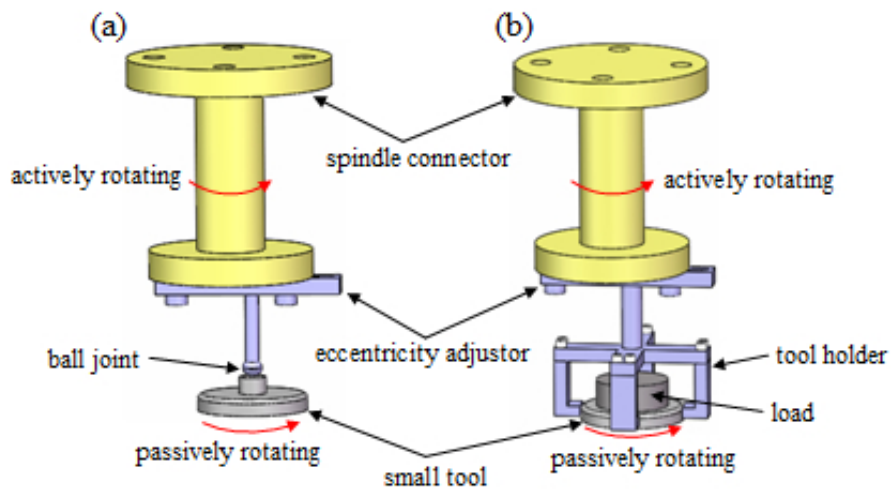

FIG. 8 Schematic of the small tool heads. (a) tool driven by ball joint, (b) tool driven by holder.

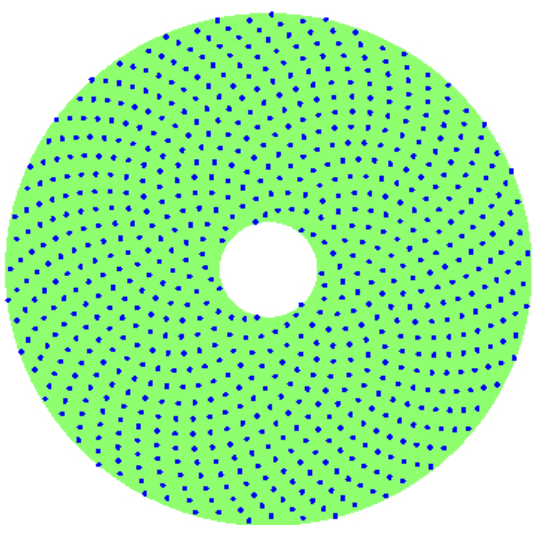

FIG. 9 Dwell points generated by 'uniform-area-increment' type spiral path.

item $(\theta)$ represents the polar angle and the second $(r)$ the polar axis. The angle increment $(\varphi)$ and the spiral path coefficient (b) should be set properly. Dwell time at each dwell point is the product of the dwell time density at the point and the area enclosed by the point, of which the former can be obtained by various algorithms $[27,28]$ and the later by Eq. (8). Dwell time at local dwell points (e.g.P $(i))$ is implemented during the path interval from $P(i-1 / 2)$ (the middle location between $P(i-1)$ and $P(i))$ to $P(i+1 / 2)$. During this path interval, the angular velocity of the pad is set $\varphi / t(i)$ and the linear velocity $(r(i+1 / 2)-r(i-1 / 2)) / t(i)$. A servo motor system is required for the full-aperture polisher to control the angular position and velocity of pad. The polishing machine using polyurethane pad is mostly equipped with a workpiece head which controls the translation of the workpiece on pad. For the machine of this type, the workpiece head can be used to fix the tool head and control the linear position and velocity of the tool. As for the conventional continuous polishing machines without such a workpiece head, a guide and movable block equipped with a servo motor as proposed above for the measurement of the pad shape has to be installed. Finally, the code for controlling the movement of the tool head is generated accordingly and the pad shape correction process is conducted.

$$
\begin{gathered}
P(i):\left\{\begin{array}{l}
\theta(i)=i \varphi \\
r(i)=b(i \varphi)^{1 / 2}
\end{array}\right. \\
\left\{\begin{array}{l}
C(i)=(2 i-1) b^{2} \varphi / 4, \quad i \varphi \leq 2 \pi \\
C(i)=\pi b^{2} \varphi, \quad i \varphi>2 \pi
\end{array}\right.
\end{gathered}
$$




\section{EXPERIMENTAL VALIDATIONS}

\subsection{Experimental configuration}

The experiments were conducted on a full-aperture polisher with a polyurethane pad. The polisher is equipped with a marble platen, a high precision guide and a workpiece spindle. The platen has a size of $1100 \mathrm{~mm}$ in diameter and $400 \mathrm{~mm}$ in thickness. Angular position and velocity of the platen is controlled by a servo motor. A polyurethane pad (GR-35 by Universal Photonics Inc.; $1.27 \mathrm{~mm}$ thick with square patterned grooves (1 mm width, $10 \mathrm{~mm}$ spacing)) is adhered to the platen. The guide is installed above the platen, and it is parallel to the platen along the diametric direction. A movable block driven by a servo motor is restricted to move along the guide. A workpiece spindle is installed upright on the block. The spindle is utilized to drive the workpiece through a hold. A series of experiments have been carried out on the polisher. For simplification, in the following we only present a set of them for validation of measurement and correction of the pad shape as well as improvement of optic figure with the corrected pad. The experiments have been conducted as follows: 1) measuring the initial shape of the pad;2) polishing the optic with the pad; 3) correcting the shape of the pad; 4) polishing the optic with the corrected pad.

\subsection{Validation of the measurement methodology}

During measurement of the pad shape, the platen is rotating about its self's spindle while the displacement sensor fixed on the workpiece spindle is moving along the guide. Thus, the axial jump of the platen spindle and the guide error are the primary sources of measurement error. In order to determine the axial jump of the platen spindle while rotating, we fixed the displacement sensor on the stationary workpiece spindle and oriented it to the pad surface near the center, and then recorded the axial jump of the platen at different rotational rates $(0 \sim 20 \mathrm{RPM})$. It is revealed that the axial jump is maintained lower than $0.3 \mu \mathrm{m}$. This tiny jump error has little effect on measurement precision, so it doesn't has to be removed in practical measurement data.

The guide error is then determined with the help of a high precision mirror ( $\Phi 600 \mathrm{~mm}, \mathrm{PV}<1 \mu \mathrm{m})$. Using the method proposed in Section 2.2 we obtained the guide error in the stroke $\mathrm{x}$ : 0-500 mm with 15000 sampling points, as shown in Figure 10. This error is fairly large, thus it is important that it be subtracted from practical measurement data.

In order to validate the accuracy of the measurement methodology, we measured the surface figure of a $\Phi 500 \mathrm{~mm}$ mirror with the methodology and compared it to the shape obtained by an optical Fizeau Interferometer. Interferometer is widely used in measuring surface figure of optics because of its high precision. Herein, the surface shape of the mirror were previously determined by a $\Phi 32$ ' interferometer. During measurement with the methodology, the mirror was mount on the pad and the measuring procedure was proceeded the same as for the pad. The two shapes obtained by the Interferometer and the proposed methodology are shown in Figure 11. It can be

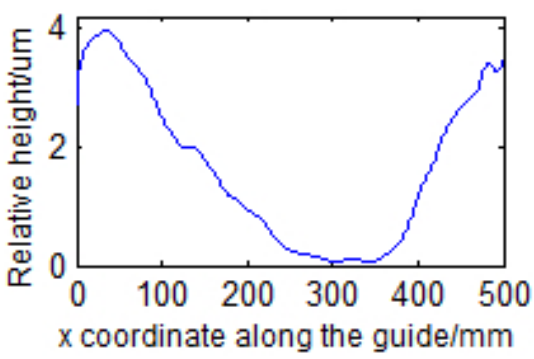

FIG. 10 Dwell points generated by 'uniform-area-increment' type spiral path.

a)
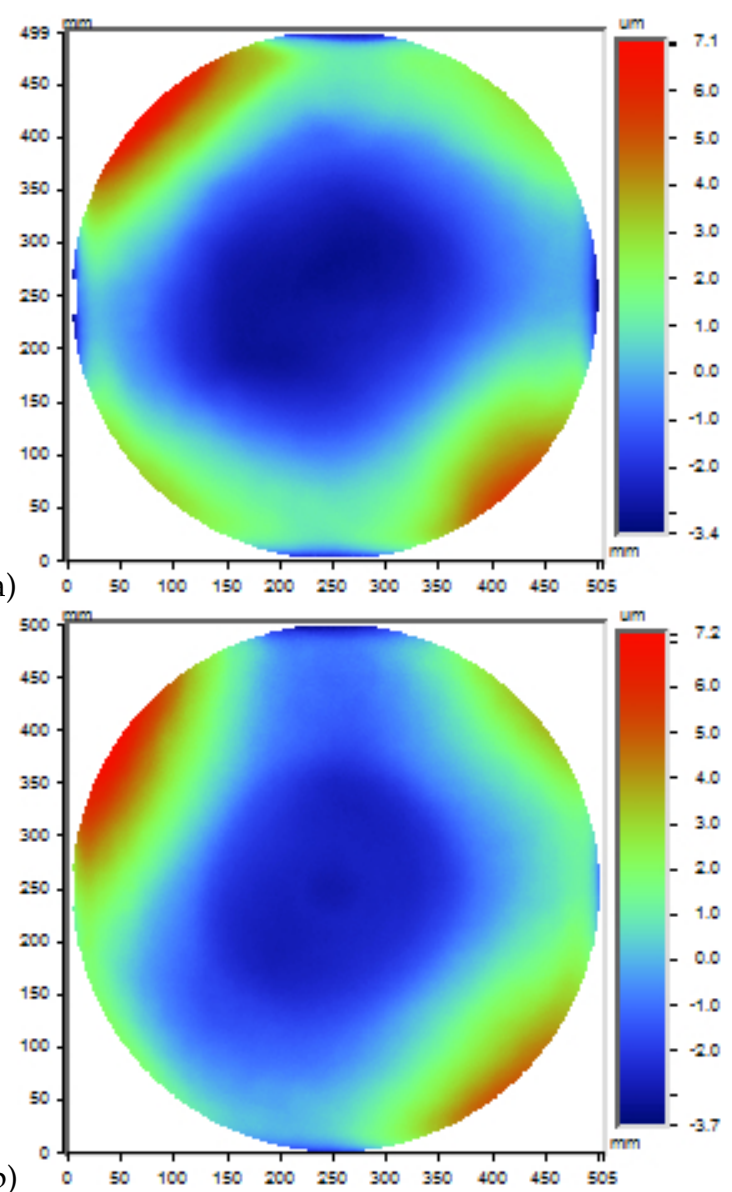

FIG. 11 Surface figures of a $\Phi 500 \mathrm{~mm}$ mirror measured by (a) interferometer and (b) the proposed methodology.

seen that the shape by the methodology is very similar with that by Interferometer, which suggests the accuracy of the proposed methodology.

\subsection{Correction of the pad shape and polishing experiments}

The experiments are shown in Figure 12. Before measuring the pad shape, we first mark out the radius at $C=0(C$ refers to the angle of the platen), as well as the inner point $\left(\mathrm{P}_{1}, \mathrm{x}=40 \mathrm{~mm}\right)$ and outer point $\left(\mathrm{P}_{2}, \mathrm{x}=540 \mathrm{~mm}\right)$. Then the platen is rotated so that the radius at $C=0$ is parallel to the guide above. The block is moved to the original position $(x=0)$ and the detecting point of the sensor orients to $P_{1}$. The linear velocity of the block is set to $25 \mathrm{~mm} / \mathrm{min}$ as it moves from the inside out; the angular velocity of the platen is set to $5 \mathrm{rpm}$ (i.e., $w=\pi / 6$ ) as it rotates clockwise. After that the block and platen are started, and the distance of the moving block and rotating pad 

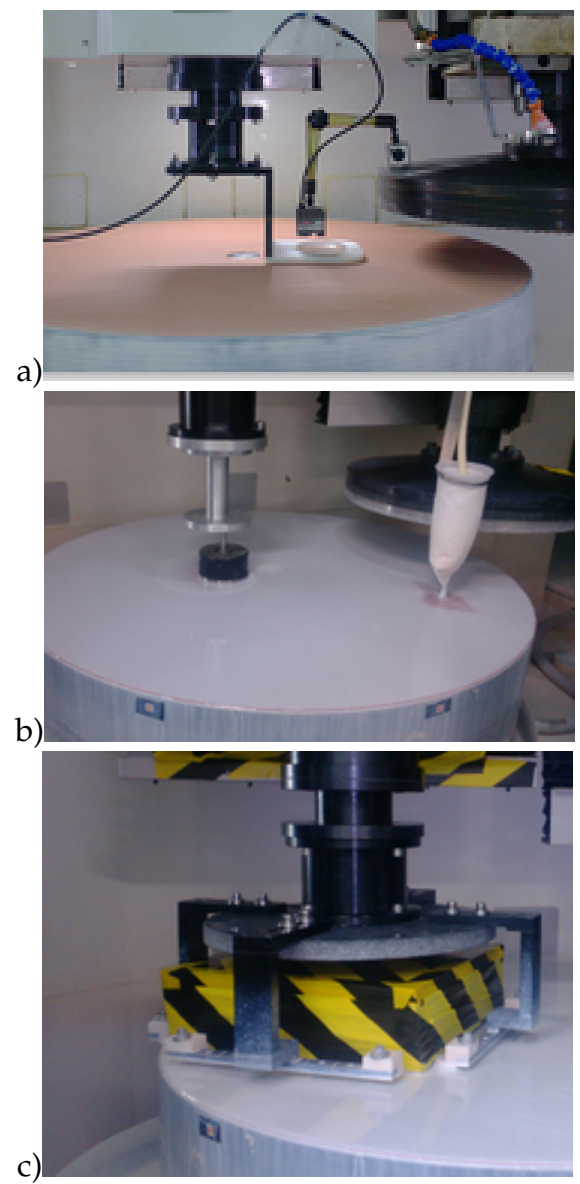

FIG. 12 Polishing experiments, (a) measuring the pad shape (b) correcting the pad shape and (c) polishing optic flat.

is simultaneously recorded by the sensor. The process takes twenty minutes. Then, we got the height of the pad at a series of spiral-distributed positions, $h(i), i=1,2, \ldots, 300000$.

The actual heights are obtained by subtracting the stroke error of the block from the detected heights, as shown in Eq. (9). Then we build a rectangular coordinate system on the pad surface. The origin locates on the center of the pad, and the $X$ axis orients from the origin to the initial detecting location (i.e. the radius at $\mathrm{C}=0) . \mathrm{x}, \mathrm{y}$ coordinates and the actual heights of the detected points can be obtained by Eq. (9). Then we generate a series of uniformly discretized points. Herein we adopt a discretized interval of $1 \mathrm{~mm}$, thus $\mathrm{x}$, y coordinates of these points can be obtained by Eq. (10) and the corresponding heights in terms of matrix represent the contour of the pad. The heights of the points within the annular pad can be solved by interpolation algorithm according the heights of the detected points, whiles those of the points located out of the annular pad is set to invalid data. The calculation takes about ten seconds in MATLAB. The obtained pad shape is shown in Figure 14.

$$
\begin{aligned}
& P(i):\left\{\begin{array}{l}
x(i)=(40+500 / 300000 \cdot i) \cos (\pi / 6 \cdot 0.004 \cdot i) \\
y(i)=(40+500 / 300000 \cdot i) \sin (\pi / 6 \cdot 0.004 \cdot i), \\
h(i)=f(i)-e(i)
\end{array}\right. \\
& i=1,2, \ldots, 300000 .
\end{aligned}
$$

$$
\begin{aligned}
& Q(k, l):\left\{\begin{array}{l}
x(k, l)=-540+l \\
y(k, l)=-k+540
\end{array}\right. \\
& k, l=1,2, \ldots 1080 .
\end{aligned}
$$

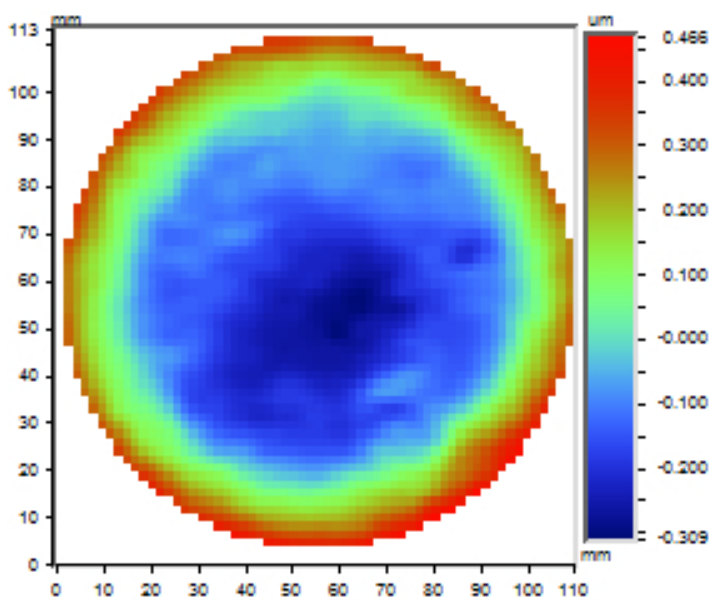

FIG. 13 Wear function obtained with $\Phi 90 \mathrm{~mm}$ diamond disc tool on the polyurethane pad. The spotting time is $1 \mathrm{~min}$.

We build a small disc tool of $\Phi 90 \mathrm{~mm}$. The diamond grits having an average size of 150 um are electroplated onto the surface of the tool. The tool is connected to the tool head through a ball joint and the entire tool head is fixed on the workpiece spindle of the polisher. The distance between the tool axis and the spindle axis (i.e. eccentricity) is set to $10 \mathrm{~mm}$. During correction of the pad shape, the tool revolves about the spindle axis driven by the spindle, and simultaneously it rotates passively about itself' axis through the ball joint. The spindle rotates at an angular speed of 80rpm, and it applies a load of $5 \mathrm{~kg}$ on the tool. We conduct a static spot experiment on the pad and the wear function obtained is shown in Figure 13. The function has a diameter of approximately $110 \mathrm{~mm}$, and the near-Gaussian shape is prone to form correction. Spiral tool path is adopted, and the dwell points are generated according to Eq. (7) [29]. Afterwards the pad shape correction process is conducted and the resultant pad shape is shown in Figure 14. The PV value of the pad is greatly reduced from $65.8 \mathrm{um}$ to $22.7 \mathrm{um}$.

Polishing experiments have been carried out before and after the pad shape correction process. The samples of fused silica flats have a size of $430 \mathrm{~mm} \times 430 \mathrm{~mm} \times 40 \mathrm{~mm}$ and are previously fine-ground. During polishing, the rotational rate of the pad is 20 RPM while that of the workpiece is 19.5 RPM. The applied pressure of the workpieces is $1.0 \mathrm{MPa}$. The spacing of the workpiece center and the pad center is $200 \mathrm{~mm}$. Each polishing experiment continues longer than 5hrs until the figure converges to a stable value, so as to eliminate the effect of the initial figure. As polishing parameters are settled at the same value in all experimenters, the resultant figure of the workpiece is mainly dependent on the pad surface shape. Surface figures of the polished flats are shown in Figure 15. It is revealed that the surface figure of the workpiece is intensively correlative to the pad shape. As the initial shape of the pad is quite convex, the polished workpiece has a fairly concave figure. After the correction process, convexity of the pad shape is markedly decreased and hence the concave figure is obviously improved. With the corrected pad, the PV value is decreased from $2.38 \lambda$ to $0.42 \lambda$ (@632.8 nm), which is superior to most other polishing results $[30,31]$. For instance, Suratwala in Lawrence Livermore National Laboratory attained 

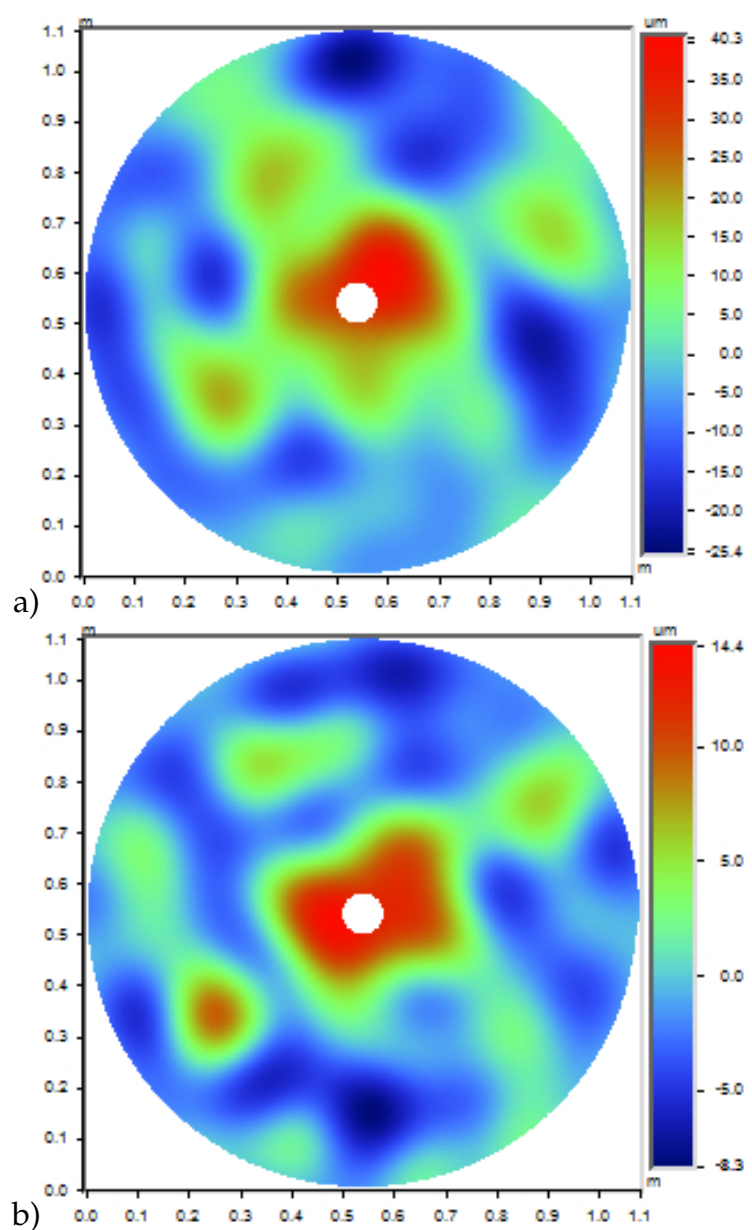

FIG. 14 Surface shape of the polyurethane pad, (a) before conditioning, PV $=65.7 \mathrm{um}$, (b) after conditioning, $\mathrm{PV}=22.7 \mathrm{um}$.

higher than $0.2 \lambda$ PV flatness on $100 \mathrm{~mm}$-sized workpieces by mitigating the non-uniform spatial material removal [31]. In summary, these experiments demonstrated that the proposed methodology is effective in correction of the pad shape, and the corrected pad is much more beneficial to improve the figure precision of the flats.

\section{CONCLUSIONS}

A novel method was developed to measure the pad shape. The method first gets the height of the pad at spirally distributed locations detected by the translating laser displacement sensor above the rotating pad, and then the pad shape in terms of height matrix is calculated by interpolation algorithm based on the detected spirally distributed points. Also, a method for deterministic correction of the pad shape based on a small tool was proposed. With this method the pad shape is corrected by controlling the dwell time map of the tool on the pad surface in a spiral tool path. The small tool constructed with diamond-electroplated disc has been proven to be effective to produce a well distributed wear function on the polyurethane pad. In the experiments conducted on a full aperture polisher with a polyurethane pad, the pad shape was accurately generated and deterministic correction of the pad shape has been validated. The methods proposed here maybe extended to pitch pad in other full aperture polishing processes.
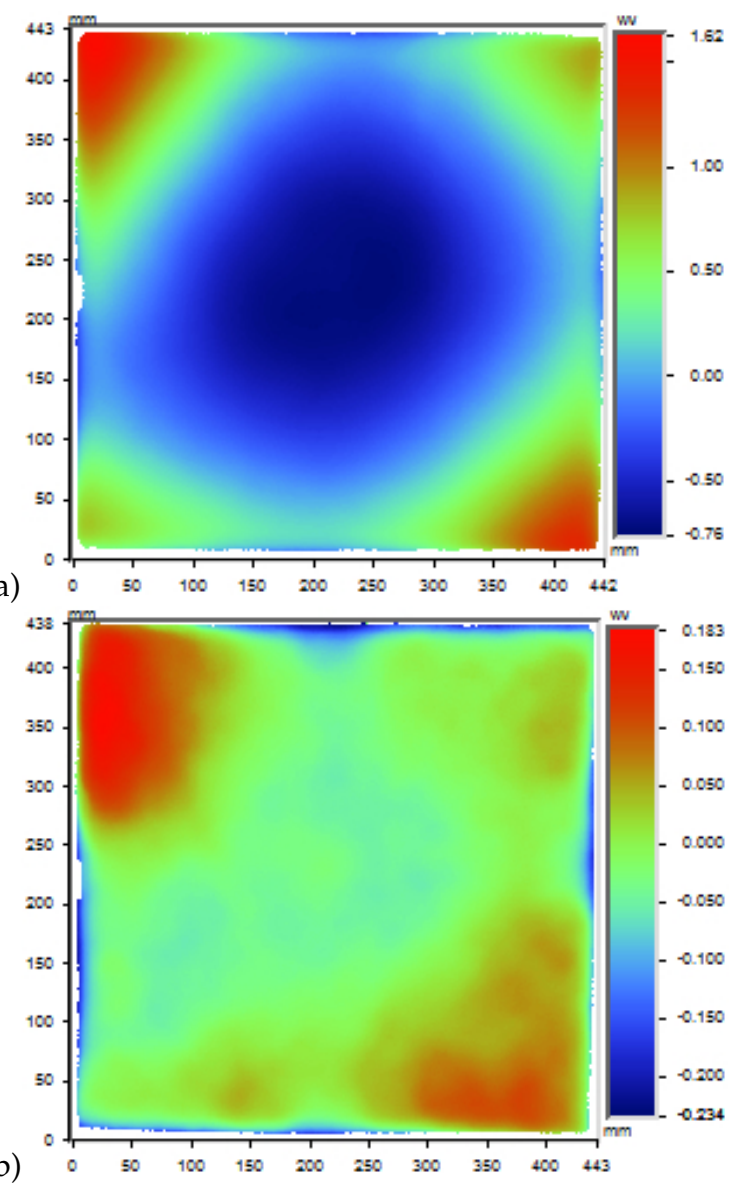

FIC. 15 Surface figure of the polished optic, (a) with the pad before conditioning, $\mathrm{PV}=2.38 \lambda$, (b) with the pad after conditioning, $\mathrm{PV}=0.42 \lambda$.

\section{References}

[1] J. K. Lawson, J. M. Auerbach, R. E. English, M. A. Henesian, J. T. Hunt, R. A. Sacks, J. B. Trenholme, et al., "NIF Optical Specifications - The Importance of the RMS Gradient," Proc. SPIE 3492, 336 (1998).

[2] H. Y. Tam, and H. B. Cheng, "An investigation of the effects of the tool path on the removal of material in polishing," J. Mater. Process. Tech. 210(5), 807-818 (2010).

[3] J. M. Tamkin, and T. D. Milster, "Effects of structured mid-spatial frequency surface errors on image performance," Appl. Optics 49(33), 6522-6536 (2010).

[4] D. Liao, Z. Yuan, C. Tang, R. Xie, and X. Chen, "Mid-Spatial Frequency Error (PSD-2) of optics induced during CCOS and fullaperture polishing," J. Eur. Opt. Soc.-Rapid 8, 13031 (2013).

[5] T. I. Suratwala, M. D. Feit, and W. A. Steele, "Material removal and surface figure during pad polishing of fused silica," J. Am. Ceram. Soc. 93(5), 1326-1340 (2010).

[6] F. Cooke, N. Brown, and E. Prochnow, "Annular lapping of precision optical flatware," Opt. Eng. 15(5), 155407-155407 (1976).

[7] D. W. Kim, S. W. Kim, and J. H. Burge, "Non-sequential optimization technique for a computer controlled optical surfacing process using multiple tool influence functions," Opt. Express 17(24), 21850-21866 (2009).

[8] C. Fan, J. Zhao, L. Zhang, Y. S. Wong, G. S. Hong, and W. S. Zhou, "Modeling and analysis of the material removal profile for free abrasive polishing with sub-aperture pad," J. Mater. Process. Tech. 214(2), 285-294 (2014). 
[9] J. C. Lambropoulos, C. Miao, and S. D. Jacobs, "Magnetic Field Effects on Shear and Normal Stresses in Magnetorheological Finishing," Opt. Express 18(19), 19713-19723 (2010).

[10] J. Arkwright, J. Burke, and M. Gross, "A deterministic optical figure correction technique that preserves precision-polished surface quality," Opt. Express 16(18), 13901-13907 (2008).

[11] T. Wang, H. B. Cheng, Z. C. Dong, and H. Y. Tam, "Removal character of vertical jet polishing with eccentric rotation motion using magnetorheological fluid," J. Mater. Process. Tech. 213(9), 15321537 (2013).

[12] Z. Z. Wang, R. Pan, Y. B. Guo, D. X. Zhang, Y. H. Xie, and J. Wang, "Controllability of stiffness of bonnet tool polishing larges aspheric lenses," High power laser and particle beams 25(9), 2270-2274 (2013).

[13] K. Park, J. Park, B. Park, and H. Jeong, “Correlation between breakin characteristics and pad surface conditions in silicon wafer polishing," J. Mater. Process. Tech. 205(1)-(3), 360-365 (2008).

[14] D. Liao, J. Wang, S. Zhao, R. Xie, X. Chen, Z. Yuan, B. Zhong, X. Xu, and S. Zhang, "Analysis of the optic/impurity-particle/pad interaction for reduction of scratches formed on optics during pad polishing," J. Non-Cryst. Solids 391, 96-100 (2014).

[15] F. W. Preston, "The theory and design of plate glass polishing machines," J. Soc. Glass Tech. 11, 214-256 (1927).

[16] D. Liao, R. Xie, J. Hou, X. Chen, and B. Zhong, "A polishing process for nonlinear optical crystal flats based on an annular polyurethane pad," Appl. Surf. Sci. 258(22), 8552-8557 (2012).

[17] S. R. Runnels, I. Kim, J. Schleuter, C. Karlsrud, and M. Desai, “Modelling tool for chemical- mechanical polishing design and evaluation," IEEE T. Semiconduct. M. 11(3), 501-510 (1998).

[18] Y. B. Xin, "Modeling of pad-wafer contact pressure distribution in chemical-mechanical polishing," Int. J. Mfg. Sci. Technol. 2(1), 20-33 (2000).

[19] G. Fu, and A. Chandra, "A model for wafer scale variation of material removal rate in chemical mechanical polishing based on viscoelastic pad deformation," J. Electron. Mater. 31(10), 1066-1073 (2002).

[20] 0. Chang, H. Kim, K. Park, B. Park, H. Seo, and H. Jeong, "Mathematical modeling of CMP conditioning process," Microelectron. Eng. 84(4), 577-583 (2007).
[21] Y. Y. Zhou, and E. C. Davis, "Variation of polish pad shape during pad dressing," Mater. Sci. Eng. B 68(2), 91-98 (1999).

[22] Y. G. Wang, Y. W. Zhao, and J. Gu, "A new nonlinear-microcontact model for single particle in the chemical-mechanical polishing with soft pad," J. Mater. Process. Tech. 183(2)-(3), 374-379 (2007).

[23] D. Liao, X. Chen, C. Tang, R. Xie, and Z. Zhang, “Characteristics of hydrolyzed layer and contamination on fused silica induced during polishing," Ceram. Int. 40(3), 4479-4483 (2014).

[24] M. Y. Tsai, and Y. S. Liao, "Dressing characteristics of oriented single diamond on CMP polyurethane pad," Mach. Sci. Technol. 13(1), 92-105 (2009).

[25] D. W. Kim, and S. W. Kim, "Static tool influence function for fabrication simulation of hexagonal mirror segments for extremely large telescopes," Opt. Express 13(3), 910-917 (2005).

[26] L. Zhou, Y. Dai, X. Xie, and S. Li, “A Novel Path Used in Computercontrolled Polishing Process Based on Uniform-area-increment Spiral," Journal of National University of Defense Technology 31(4), 1-4 (2009).

[27] C. Song, Y. Dai, and X. Peng, "Model and algorithm based on accurate realization of dwell time in magnetorheological finishing," Appl. Optics 49(19), 3676-3683 (2010).

[28] H. Y. Tam, O. C. Lui, and C. K. Mok, "Robotic polishing of free-form surfaces using scanning paths," J. Mater. Process. Tech. 95(1)-(3), 191-200 (1999).

[29] C. Wang, W. Yang, Z. Wang, X. Yang, C. Hu, B. Zhong, Y. Guo and Q. Xu, "Dwell-time algorithm for polishing large optics," Appl. Optics 53(21), 4752-4760 (2014).

[30] D. F. Liao, H. Zhao, Z. G. Yuan, and R. Q. Xie, “Improvement of Surface Figure in the Polyurethane Pad Continuous Polishing Process," Appl. Mech. Mater. 319, 107-112 (2013).

[31] T. Suratwala, R. Steele, M. Feit, R. Desjardin, and D. Mason, "Convergent Pad Polishing of Amorphous Silica," International Journal of Applied Glass Science 3(1), 14-28 (2012). 